\title{
Disseminated Superficial Actinic Porokeratosis on the Face Treated with Imiquimod $5 \%$ Cream
}

\author{
Hassan Riad Khaled Mansour Hussein Al Sada Samya Abu Shaika \\ Hamda Al Ansari Haya Al Mohannadi \\ Rumailah Hospital, Doha, Qatar
}

\section{Key Words}

Porokeratosis · Facial lesions · Imiquimod cream

\begin{abstract}
Porokeratoses are a group of hereditary or acquired disorders characterized by annular lesions with an atrophic center and a prominent peripheral ridge. Pathologically, porokeratosis is characterized by the presence of abnormal clones of keratinocytes that form a column of parakeratotic cells, called the cornoid lamella. Fifteen percent of patients of disseminated superficial actinic porokeratosis (DSAP) have facial lesions; other regions like the extensor surface of extremities constitute the majority of reported cases. Exclusively facial lesions are probably less frequent. Rarely, actinic porokeratosis is confined to the nose only. Of reported porokeratosis cases, $7.5 \%$ have revealed a malignancy arising within the lesion. We present a case of facial sporadic DSAP that was treated with imiquimod $5 \%$ cream in conjunction with a regular sunscreen. Follow-up of this case is important to rule out the possibility of malignant transformation of the lesions.

(c) 2013 S. Karger AG, Basel

Porokeratoses are a group of hereditary or acquired disorders characterized by annular lesions with an atrophic center and a prominent peripheral ridge. Pathologically, porokeratosis is characterized by the presence of abnormal vacuolated keratinocyte clones that develop in between normal cells, with no granules, and which eventually form a column of parakeratotic cells, a rim called the cornoid lamella. Since its first description by Mibelli and Respighi in 1893, hundreds of cases have been published. There are at least six clinical types of porokeratosis, namely: classic porokeratosis of Mibelli, linear porokeratosis, disseminated superficial actinic porokeratosis (DSAP), disseminated superficial porokeratosis, porokeratosis palmaris et plantaris disseminate and porokeratosis palmaris plantaris punctata [1].

Other atypical types are facial and giant porokeratosis, porokeratosis ptychotropica and porokeratoma. In addition, there are the rare forms of porokeratosis, i.e. porokeratotic
\end{abstract}


Riad et al.: Disseminated Superficial Actinic Porokeratosis on the Face Treated with Imiquimod $5 \%$ Cream

adnexal ostial nevus, porokeratotic eccrine ostial and dermal duct nevus and porokeratotic eccrine and hair follicle nevus. Other forms are punched-out, hypertrophic verrucous and reticulate porokeratosis [2,3]. We report a case of DSAP that presented on the face and was treated successfully with imiquimod.

\section{Case Report}

A 19-year-old, single, unemployed, Caucasian female (skin type III) presented with multiple facial lesions. She had been suffering from multiple pigmented annular papules and plaques on her face for 7 years. The lesions were slowly progressive with no signs of selfhealing. She had been a normal, healthy, full-term baby and had normal physical milestones of development as a child. She appeared to be immunocompetent with no history of recurrent infections or any other skin lesions. Her parents and siblings $(3$ brothers and 2 sisters) were all normal and healthy. She had no family history of a similar condition or other skin problems. Her medical history was unremarkable with no other obvious complaints, and she was not overexposed to sunlight as she spent most of her time indoors. Upon examination, the central part of the face, i.e. the malar area and nose including the ala nasi, showed scattered annular papules and plaques of different sizes ranging from 0.3 to $2 \mathrm{~cm}$. The lesions were asymptomatic and symmetrically distributed on both sides of the face (figs. $1,2)$. Routine laboratory tests were done and the results were within normal ranges. Multiple biopsies were taken from different lesions. The diagnosis was confirmed as DSAP, with a classic and very illustrative pathological picture (figs. 3-6). Treatment options were multiple, though none was considered an obvious first choice. All physical modalities were excluded to avoid the possibility of side effects like scarring and disfigurement, as the lesions were located in the central part of the face and involved the skin overlying the nasal cartilage. We first prescribed a topical retinoic acid $0.1 \%$ cream that has keratolytic and antineoplastic effects, and it was assumed that this might rectify the faulty clonal epidermopoiesis. The patient applied the cream twice daily for 3 months without a significant response. Six months later, the patient presented again and the picture was almost unchanged except for a few small new lesions in the malar area and on the nose. We decided to give her imiquimod 5\% cream to be used once a day (3 times per week) for 24 weeks. She came for a follow-up every 2 weeks. Her skin started to respond after 1 month. Side effects like erythema, crustations and pruritus were experienced and were controlled by topical emollient cream; we avoided corticosteroids and calcineurin inhibitors because they can counter the effects of imiquimod. On completion of the therapy, the response was very satisfactory for both the patient and the therapist. There were some lesions with only a partial response. Central scarring and the scars across the nose improved in their color, thickness and texture, constituting an added benefit of imiquimod in this indication. The patient maintained a once-weekly application of imiquimod. There has been no relapse after follow-up for 2 years now.

\section{Discussion}

Porokeratosis is inherited as an autosomal dominant trait; however, sporadic cases are also known to occur. Chernosky and Freeman [4] first described DSAP in 1967 in a Texas population. DSAP is the most common of the clinical variants and may account for almost half of all cases in the USA [5], while in Singapore it is second to the Mibelli type in frequency 
Riad et al.: Disseminated Superficial Actinic Porokeratosis on the Face Treated with Imiquimod 5\% Cream

(18 and 56\%, respectively) [6]. It usually develops in the third and fourth decades of life. The incidence in females is double that of males, unlike the classic Mibelli type. DSAP is characterized by multiple lesions that are superficial, relatively smaller than the Mibelli type, slightly pigmented, annular, keratotic and with a central atrophic area. It mainly affects areas exposed to sunlight; however, paradoxically, the limbs are more affected than the face [7].

Fifteen percent of DSAP patients have lesions on the face. Other regions such as the extensor surfaces of the extremities constitute the majority of reported cases, with cases of lesions appearing exclusively on the face being probably less frequent. Rarely, actinic porokeratosis is confined to the nose only. Of cases reported, $7.5 \%$ have revealed a malignancy arising within the porokeratosis. Unlike other forms, DSAP does not appear to have more risk of malignant change than other types [8]. It can sometimes coexist with other forms of porokeratosis (Mibelli type, linear porokeratosis, porokeratosis palmaris et plantaris disseminata and punctate porokeratosis) [9]. There is good evidence that ultraviolet light can precipitate the development of new lesions or exacerbate pre-existing lesions [5]. Our patient was minimally exposed to the sun and she spent most of her time indoors.

The exact pathogenesis of porokeratosis is not clear; however, it has been assumed that a focal clonal expansion of abnormal cells gives rise to the shape of the coronoid lamella. The coexistence of different variants in one patient or in several members of an affected family indicates different phenotypic expressions of a common genetic entity which could possibly be explained by a simultaneous expression of closely linked genes.

Genetic studies have mapped the loci responsible for DSAP to chromosomes 12q, 15q and 18q. Several mutations have been identified in the SSH1 gene on chromosome 12, which encodes a phosphatase that plays a pivotal role in actin dynamics $[10,11]$. In a genome-wide scanning and linkage analysis performed on six generations of a Chinese family with DSAP, two missense mutations in SSH1 and ARPC3 were found, which are involved in the actin cytoskeleton pathway and interact with adherent junctions in the epidermal cells. This finding suggested that cytoskeleton disorganization in epidermal cells was likely associated with the pathogenesis of DSAP [12]. In a recent study on four Chinese families, in 33\% of the patients, three mutations were found in the mevalonate kinase (MVK) gene which plays a role in lipid metabolism [13].

Psoriasis and phototherapy (UVA, BB-UVB and NB-UVB) have been associated with porokeratosis. DSAP has also been associated with immune suppression, HIV infection, diabetes mellitus, liver cirrhosis, acute pancreatitis, Crohn's disease, solid malignancy, the administration of immunomodulating drugs used to treat autoimmune diseases and following the transplantation of organs (particularly in kidney transplant patients) [14,15].

Treatment of DSAP is often difficult. Therapies such as cryotherapy, photodynamic therapy, erbium YAG and $\mathrm{CO}_{2}$ lasers or the application of topical 3\% diclofenac gel or 5fluorouracil cream are usually partially successful, but with inconsistent results [16]. Topical imiquimod 5\% cream may prove to be a useful treatment option for DSAP [17]. This therapy treated the plaques effectively, there were no more keratotic elevations or furrows, and even the central scars showed an improvement in color and texture. Biologically, imiquimod is also antineoplastic and is used to treat nonmelanoma skin cancers; this is a major added advantage because DSAP may, in rare instances, be complicated by squamous cell carcinoma. Further studies are required to confirm whether imiquimod should have a place in the management of DSAP. The mechanism of action of imiquimod in the treatment of DSAP is not yet clear: it may suppress or switch off the abnormal mutant genes through its immunological effects on both adaptive and innate immunity. 
Riad et al.: Disseminated Superficial Actinic Porokeratosis on the Face Treated with Imiquimod 5\% Cream

In conclusion, we presented a case of facial sporadic DSAP, which was treated by application of imiquimod 5\% cream in conjunction with regular sunscreens. Follow-up of this case is important to rule out the possibility of malignant transformation.

\section{References}

1 Niimi Y, Kawana S: Type 2 segmental manifestation of disseminated superficial actinic porokeratosis in a 7year-old girl. Eur J Dermatol 2009;19:25-28.

-2 Goddard DS, Rogers M, Frieden IJ, Krol AL, White CR Jr, Jayaraman AG, Robinson-Bostom L, Bruckner AL Ruben BS: Widespread porokeratotic adnexal ostial nevus: clinical features and proposal of a new name unifying porokeratotic eccrine ostial and dermal duct nevus and porokeratotic eccrine and hair follicle nevus. J Am Acad Dermatol 2009;61:1060.e1-e14.

-3 Criscione V, Lachiewicz A, Robinson-Bostom L, Grenier N, Dill SW: Porokeratotic eccrine duct and hair follicle nevus (PEHFN) associated with keratitis-ichthyosis-deafness (KID) syndrome. Pediatr Dermatol 2010;27:514-517.

4 Chernosky ME, Freeman RG: Disseminated superficial actinic porokeratosis (DSAP). Arch Dermatol 1967;96:611-624.

5 Mehta V, Balachandran C: Simultaneous co-occurrence of porokeratosis of Mibelli with disseminated superficial actinic porokeratosis. Indian J Dermatol 2009;54:390-391.

6 Tan LS, Chong WS: Porokeratosis in Singapore: an Asian perspective. Australas J Dermatol 2012;53:e40e44.

7 Khaled A, Kourda M, Abdelmoula F, M'ssedi L, Tougourti MN, Kamoun MR: Late-onset disseminated superficial actinic porokeratosis in an elderly woman. Dermatol Ther (Heidelb) 2011;1:15-19.

-8 Lee H R, Han TY, Son S-J, Lee JH: Squamous cell carcinoma developing within lesions of disseminated superficial actinic porokeratosis. Ann Dermatol 2011;23:536-538.

-9 Murase J, Gilliam AC: Disseminated superficial actinic porokeratosis co-existing with linear and verrucous porokeratosis in an elderly woman: update on the genetics and clinical expression of porokeratosis. J Am Acad Dermatol 2010;63:886-891.

10 Zhang Z, Niu Z, Yuan W, Liu W, Xiang L, Zhang J, Chu X, Zhao J, Jiang F, Chai B, Cui F, Wang Y, Zhang K, Wang Y, Xu S, Xia L, Gu J, Zhang S, Meng X, Wang S, Gao S, Fan M, Nie L, Zheng Z, Huang W: Fine mapping and identification of a candidate gene SSH1 in disseminated superficial actinic porokeratosis. Hum Mutat 2004;24:438.

11 Xia JH, Yang YF, Deng H, Tang BS, Tang DS, He YG, et al: Identification of a locus for disseminated superficial actinic porokeratosis at chromosome 12q 23.2-24.1. J Invest Dermatol 2000;114:1071-1074.

$\checkmark 12$ Zhang ZH, Huang W, Niu ZM, Liu WD, Xiang LH, Yuan WT, Zhao JJ, Gu CY, Chai B, Jiang FX, Zhang J, Xu SJ, Zheng ZZ: Two closely linked variations in actin cytoskeleton pathway in a Chinese pedigree with disseminated superficial actinic porokeratosis. J Am Acad Dermatol 2005;52:972-976.

-13 Zhou Y, Liu J, Fu X, Yu Y, Shi B, Yu G, Shi Z, Wu W, Pan F, Tian H, Liu H, Zhang F: Identification of three novel frameshift mutations of the MVK gene in four Chinese families with disseminated superficial actinic porokeratosis. Br J Dermatol 2013;169:193-195.

14 Kawara S, Oiso N, Kawada A: Disseminated superficial actinic porokeratosis in a patient undergoing treatment with long-term narrowband ultraviolet B for psoriasis. J Dermatol 2011;38:585-587.

15 Kanitakis J, Euvrard S, Faure M, Claudy A: Porokeratosis and immunosuppression. Eur J Dermatol 1998;8:459-465.

$\checkmark 16$ Chrastil B, Glaich AS, Goldberg LH, Friedman PM: Fractional photothermolysis: a novel treatment for disseminated superficial actinic porokeratosis. Arch Dermatol 2007;143:1450-1452.

17 Arun B, Pearson J, Chalmers R: Disseminated superficial actinic porokeratosis treated effectively with topical imiquimod 5\% cream. Clin Exp Dermatol 2011;36:509-511. 
Riad et al.: Disseminated Superficial Actinic Porokeratosis on the Face Treated with Imiquimod $5 \%$ Cream

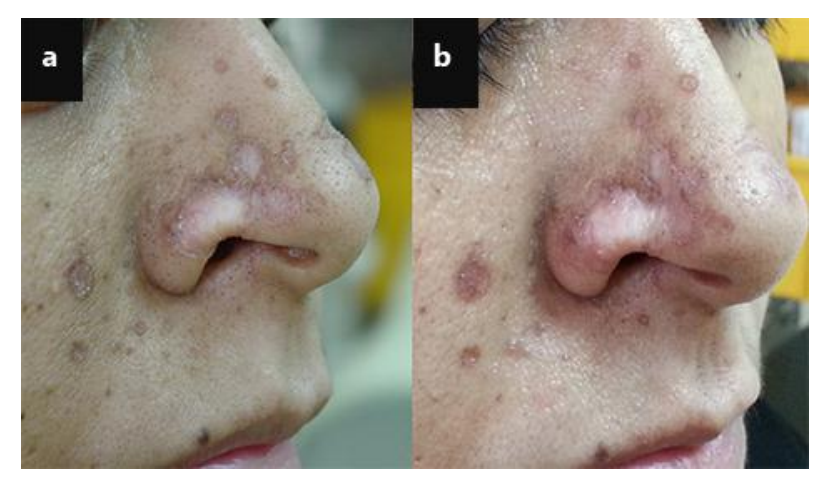

Fig. 1. The right side of the patient's face before (a) and after (b) imiquimod $5 \%$ cream therapy (once a day, 3 times weekly for 16 weeks). Note the disappearance of the keratotic rim, the flattening of the lesions and the improvement in the color and thickness of the scars. Residual hyperpigmentation and hypopigmentation remained unchanged.

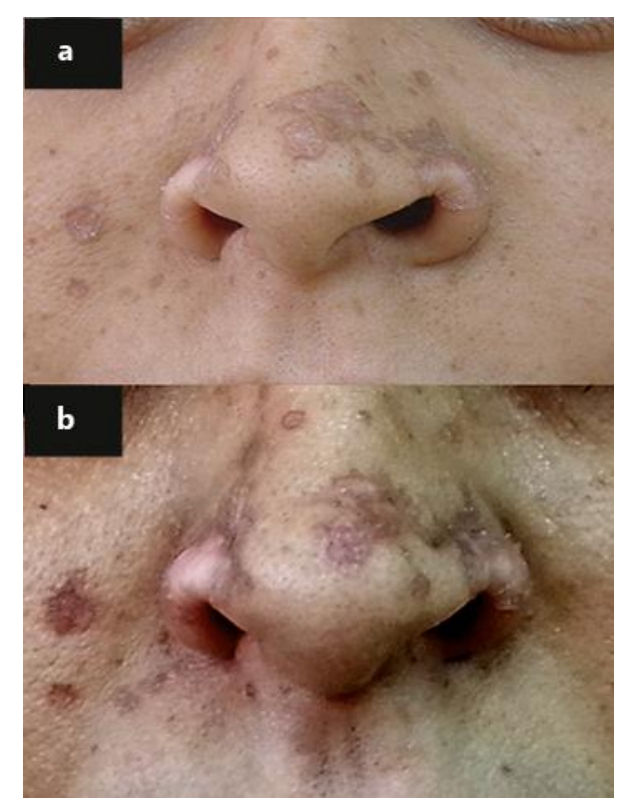

Fig. 2. The patient before (a) and after (b) imiquimod 5\% cream therapy (once a day, 3 times weekly for 16 weeks). Notice the flattening of the lesions and the improvement of skin texture. 
Riad et al.: Disseminated Superficial Actinic Porokeratosis on the Face Treated with Imiquimod 5\% Cream
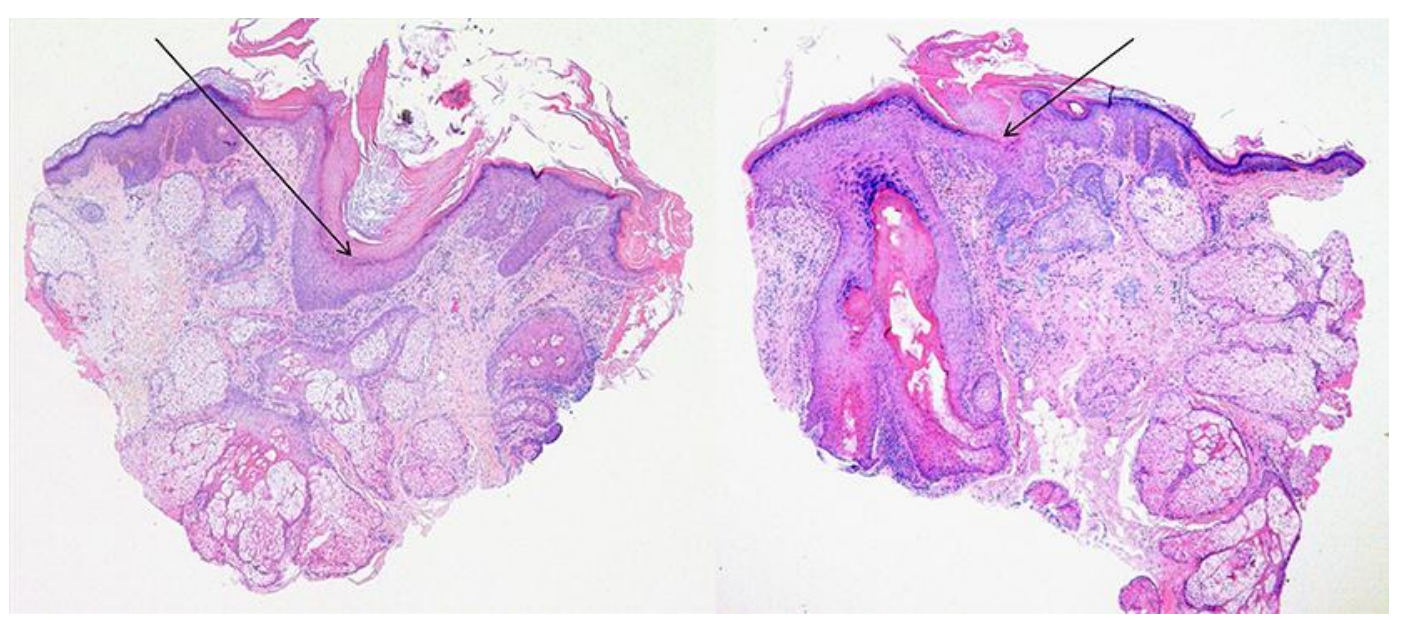

Fig. 3. A histopathology section. $\times 100$. Black arrows point to invaginations in the epidermis of both specimens. Note the outward projection of a parakeratotic column called the cornoid lamella. This is a classic, characteristic picture of porokeratosis.

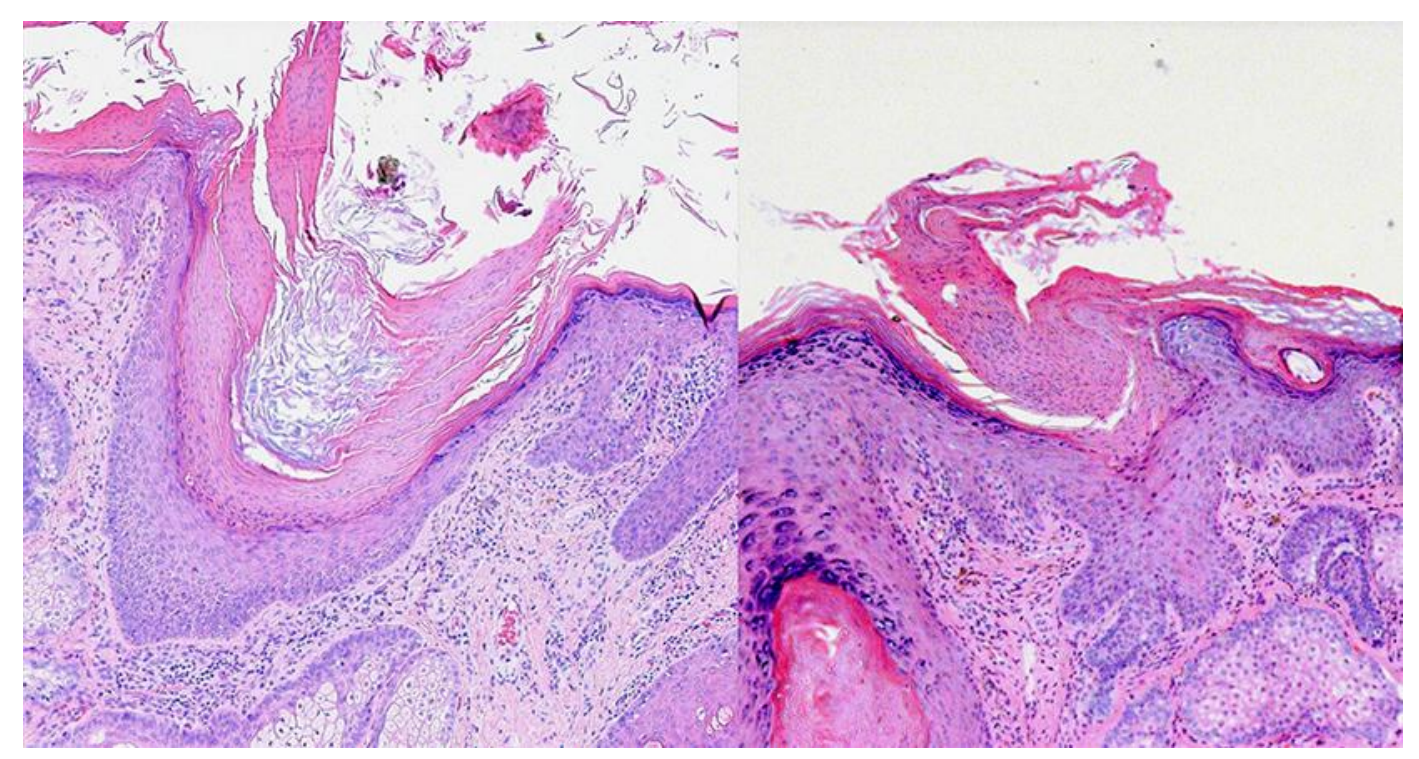

Fig. 4. A histopathology section. $\times 200$. A closer view of the pathognomonic features of porokeratosis. 
Riad et al.: Disseminated Superficial Actinic Porokeratosis on the Face Treated with Imiquimod $5 \%$ Cream

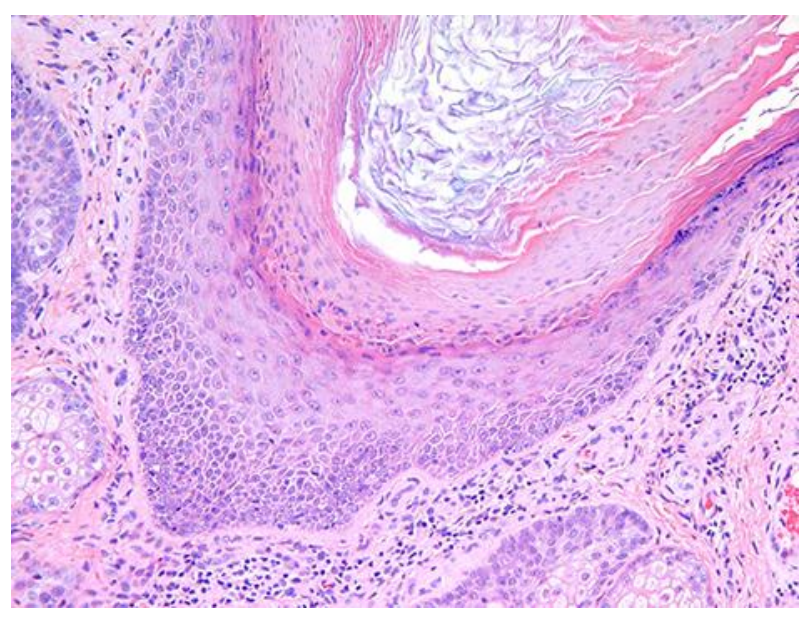

Fig. 5. A histopathology section. $\times 400$. A section showing abnormal vacuolated keratinocyte clones that develop in between normal cells and interrupt the granular layer, with no granules in the abnormal cells. A column of parakeratotic cells forms, called the cornoid lamella.

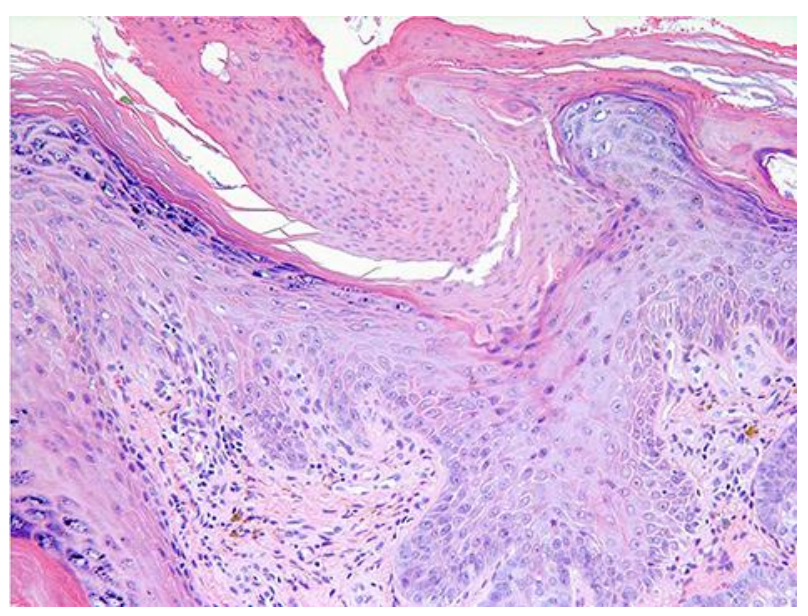

Fig. 6. A histopathology section. $\times 400$. Another lesion with similar features to figure 5 . 